\title{
Magnetic dichroism and spin-resolved photoemission from rough interfaces
}

\author{
V. M. Uzdin, ${ }^{*}$ D. Knabben, F. U. Hillebrecht, and E. Kisker \\ Institute für Angewandte Physik, Heinrich-Heine-Universität Düsseldorf, D-40225 Düsseldorf, Germany
}

(Received 22 June 1998)

\begin{abstract}
The magnetic structure of ultrathin $\mathrm{Cr}$ films on $\mathrm{Fe}$ is analyzed by taking into account the roughness of the surface and of the interface. An algorithm for the epitaxial growth of the film simulates the near-surface structure, and the magnetic moment distribution is calculated self-consistently in a periodic Anderson model. For rough interfaces we find that the layered antiferromagnetic structure of the $\mathrm{Cr}$ adlayer is quenched. Within this model we determine the spin polarization and the value of the magnetic linear dichroism to be expected in angle-resolved photoemission. Comparing with experimental data it is concluded that $\mathrm{Cr}$ grows on the $\mathrm{Fe}(001)$ in a Stranski-Kastranoff mode. We propose an explanation based on the confinement of the itinerant electrons within the Cr islands on the Fe substrate. [S0163-1829(99)13801-3]
\end{abstract}

\section{INTRODUCTION}

Low-dimensional magnetic structures (LDMS) are of interest both for technical applications in magnetic data storage systems as well as in their own right. Exchange coupling between two ferromagnetic films via a nonmagnetic interlayer is a key ingredient in such systems. For optimum performance of these systems it is desirable to control the magnetic properties at the interface. In the case of a genuinely nonmagnetic interlayer, the influence of the interlayer on the magnetic moments in the ferromagnetic (FM) layer near to the interface is of prime interest. In this case there may be magnetic moments induced on the atoms in the interlayer by the interaction with the ferromagnetic layer.

A classical example of a system where the interface electronic and magnetic structure was investigated thoroughly by various methods are $\mathrm{Fe} / \mathrm{Cr}$ multilayers and $\mathrm{Cr}$ adlayers on Fe. ${ }^{1-7}$ The general problem with the interpretation of all experiments is connected to the detailed structural properties of the interface. Electronic structure calculations consistently yield a very large magnetic moment, of the order of $2.5 \mu_{B}$, for a $\mathrm{Cr}$ monolayer on $\mathrm{Fe}$. However, to date the experimental evidence for such a large moment is not conclusive. While spectroscopic experiments have not shown a large $\mathrm{Cr}$ magnetic moment, ${ }^{1,2}$ a large $\mathrm{Cr}$ moment was apparently present in the experiments by Turtur and Bayreuther. ${ }^{4}$ The short period oscillation of the interlayer coupling as observed for $\mathrm{Fe} / \mathrm{Cr} / \mathrm{Fe}$ trilayers grown on $\mathrm{Fe}$ whiskers is in qualitative agreement with theory for perfectly smooth interfaces. Unfortunately, to date no absolute determinations of the $\mathrm{Cr}$ magnetic moments for such high-quality interfaces have been carried out. The interdependence between geometric and magnetic structures was investigated for $\mathrm{Fe} / \mathrm{Cr}$ interface by the number of experimental methods. Scanning tunneling microscopy (STM) has revealed direct information about the growth process on an atomic scale. Such experiments provide knowledge only about the surface layer, not about the surface structure on the scale of few atomic layers below the surface. Davies et al. ${ }^{8}$ found by STM for Cr overlayers on Fe that only one out of every four deposited $\mathrm{Cr}$ atoms remains in the surface layer whereas the others transfer trough the surface into the sample whereby an alloy is created in the interface region. In addition STM cannot provide data about the magnetic structure of the surface and interface region.

In magnetometer experiments ${ }^{4}$ the total magnetic moment of the sample is determined. If measurements are performed in situ during the process of deposition, this can give direct information about interface magnetism. As this method gives data concerning the total magnetic moment of the sample, the interpretation in terms of magnetization or magnetic moments associated with a specific layers is a nontrivial problem. Furthermore, continued deposition of $\mathrm{Cr}$ leads to changes of the magnetic moments of the atoms already present, and consequently it is not possible to find the $\mathrm{Cr}$ moments at an interface as a difference between moments of the sample before and after $\mathrm{Cr}$ deposition.

It is furthermore important that in $\mathrm{Cr}-\mathrm{Fe}$ systems the ad-/ interlayer atoms have sizeable magnetic moments on their own, not only because of the interaction with the Fe layer. Therefore, it is very important for an understanding of interface properties to know the magnetic moments associated with $\mathrm{Fe}$ and $\mathrm{Cr}$ atoms separately. Magnetometer data cannot provide this knowledge. Mössbauer spectroscopy gives information about the distribution of hyperfine fields (hff) and consequently about magnetic moments localized on $\mathrm{Fe}$ atoms in Fe-containing LDMS. If the sample is designed so that only an interface layers contains the ${ }^{57} \mathrm{Fe}$ isotope, whereas the other layers are grown by ${ }^{56} \mathrm{Fe},{ }^{9,10}$ then Mössbauer spectra will provide valuable knowledge about interface magnetism. The interpretation, however, of Mössbauer spectra for LDMS is again a complicated problem, because one has to take into account a nonrandom distribution of directions of magnetic moments in LDMS, ${ }^{11,12}$ as well as a contribution of the $4 s$-electron polarization, which creates a direct contact hff on the nuclei and in LDMS this may be not proportional to the local magnetic moment.

Recent investigations ${ }^{10,13}$ show that interface roughness is extremely important for the magnetic structure, and this is also reflected in the shape of the Mössbauer spectra. If for the smooth interfaces the spectrum contains distinct satellites corresponding to different local environments, a wide distribution of hff is observed for more rough interfaces such that it is impossible to separate the contributions from different configurations. Data about the distribution of magnetic mo- 
ments on the $\mathrm{Cr}$ atoms can be obtained from mössbauer spectra only indirectly through the change of hff on Fe. As a result of these various complications a general picture of interface magnetic structure appears to be very ambiguous.

Core-level photoemission has some advantages in comparison with other methods mentioned above. First, it provides information about the magnetism of $\mathrm{Fe}$ and $\mathrm{Cr}$ separately by investigating core levels with specific binding energies. ${ }^{2,14}$ Because of its high surface sensitivity, only the magnetic structure of a few surface atomic layers contributes to the signal. Furthermore, it gives information averaged over the acceptance area of the spectrometer which is large on the atomic scale. Accordingly, the overall characteristics of the sample within the probing depth of 5 to $50 \AA$ below the surface are determined.

Magnetic circular dichroism in x-ray absorption was used to study the magnetic coupling between $\mathrm{Cr}$ and $\mathrm{Fe}$ and the coverage dependence of the $\mathrm{Cr}$ net magnetic moment ${ }^{15}$ in an Fe film grown on GaAs. In these experiments, a monotonous decay of the $\mathrm{Cr}$ magnetic signal was observed, which was interpreted to result from interface roughness. In x-ray absorption, which averages over the film thickness of several monolayers, a signal oscillating between a decreasing maximum and zero should be observed if the growth mode was layer by layer. The observed monotonous decrease ${ }^{15}$ was attributed to the large roughness of the Fe substrate as inferred from scanning tunneling microscopy. This is in contrast to $\mathrm{Cr}$ films grown on surfaces of Fe whiskers. ${ }^{16}$ For such films an oscillation of the surface magnetic moment around zero was detected by polarization analysis of secondary scattered electrons, indicating a layer by layer growth on such substrates. This oscillation only starts when the coverage exceeds about three layers. However, the size of the magnetic moment could not be determined from these experiments.

In this paper we show that spectroscopic data together with a semiempirical model, which includes modeling of the deposition process together with successive self-consistent calculations in a model Hamiltonian approach of the magnetic moment distribution provide a key for the investigation of the subsurface layers on an atomic scale. An analogous theoretical model was used for the explanation of the decrease of the total magnetic moment of the $\mathrm{Fe}$ sample in the process of $\mathrm{Cr}$ covering. ${ }^{17}$ It was shown that total magnetic moment of the sample can oscillate or exponentially decrease, depending on the roughness of the interface. On the base of a similar modeling ${ }^{12}$ of a nonideal $\mathrm{Fe} / \mathrm{Cr}$ interface it was demonstrated that interface roughness is essential for an adequate description of Mössbayer spectra. The surface sensitivity of photoemission makes it necessary to develop a theory for the description of emission from the rough surfaces, which will be discussed in the next section.

As an example of the application of the theory which will be developed below we will use the data obtained for $\mathrm{Cr}$ overlayer on $\mathrm{Fe}$ using spin-resolved core-level photoemission $^{2}$ and magnetic linear dichroism in core-level photoemission. ${ }^{18}$ Spectroscopic experiments on $\mathrm{Cr}$ adlayers on Fe, e.g., spin-resolved photoemission and circular magnetic dichroism, suggest a relatively small surface $\mathrm{Cr}$ magnetic moment much less than the theoretical value $2.5 \mu_{B}$. It is plausible to attribute the discrepancy between theories for ideal interfaces and the small moments found experimentally

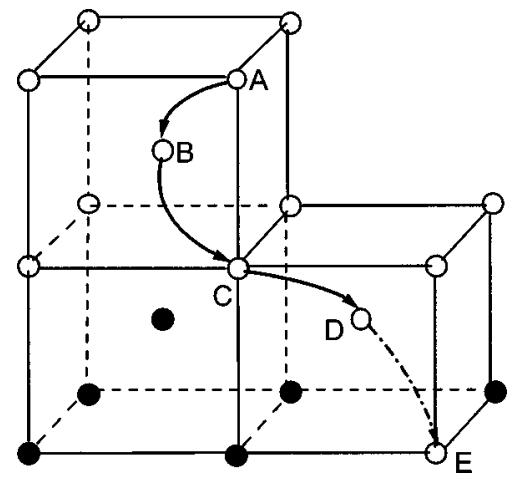

FIG. 1. Random walk of the atom through the empty sites in the bcc lattice. Solid circles correspond to filled sites. Site " $B$ ', contains four vacant neighbor sites in the next layer, and transition to any of these occurs with probability 0.25 . Site " $D$ "' has only one nearest vacant site in the next layer. For variant $a$ of the algorithm, the atom has to transfer from site $D$ to $E$. For the $b$ variant it may stay with definite probability on site $D$ so that site $E$ remains vacant as more atoms are added.

to deviations from the ideal interface in the experiments. The purpose of the present paper is to make a first step towards a more realistic description of such interfaces and the size of the net magnetic moment one may observe in a magnetically sensitive photoemission experiment. The magnetic sensitivity can either be realized by taking spin-resolved data, or by making use of (linear or circular) magnetic dichroism. Our model analysis proceeds in three steps: first, a rough surface or interface is generated by an epitaxy algorithm; second, the magnetic moments are calculated using an Anderson Hamiltonian model; and third, the magnetic signal is obtained as a weighted average of the individual magnetic moments. The weighting accounts for the attenuation of photoelectron intensity due to transmission towards and through the surface. We compare the results to experimental data on the coverage dependence of magnetic dichroism or spin-resolved photoemission. In doing so, we tacitly assume that these spectral properties can be used as a measure for the magnetic moment.

\section{MAGNETIC MOMENTS AT ROUGH INTERFACES}

To describe magnetic moments at nonideal surfaces and overlayers, we simulate this situation using the special algorithm epitaxy ${ }^{17,19}$ which allows us to create spatially inhomogeneous structures with different roughness. The algorithm fills a prism consisting of $8 \times 8 \times 18$ sites with $\mathrm{Fe}$ and/or $\mathrm{Cr}$ atoms. Outside the prism the structure is repeated periodically. Initially, the bottom layer of the prism is covered uniformly by $\mathrm{Fe}$ atoms, while all other sites are empty. The magnetic moments and $d$-electron numbers of the Fe atoms in the bottom layer are assumed to be equal to the bulk values for bcc $\mathrm{Fe}$, and are kept constant during iterations leading to self-consistency.

The epitaxy algorithm adds single Fe atoms to the top level of the prism in a random procedure and lets them descend through empty sites until further descending is blocked by occupied sites. Figure 1 illustrates the random walk of an atom in the bcc lattice. Sites which are not yet filled by atoms are depicted by empty circles. Transfer of atoms from 
TABLE I. Layer by layer distribution of $\mathrm{Fe}$ atoms, $\mathrm{Cr}$ atoms, and empty sites for a set of structures generated by the $a$ variant of algorithm "epitaxy" for different coverages.

\begin{tabular}{|c|c|c|c|c|c|c|c|c|c|c|c|c|}
\hline & & $\varsigma=\mathbf{0 , 5}$ & & & $s=\mathbf{0 , 8}$ & & & $\varsigma=1,0$ & & & & \\
\hline & $\mathrm{Fe}$ & $\mathrm{Cr}$ & Emp & $\mathrm{Fe}$ & $\mathrm{Cr}$ & Emp & $\mathrm{Fe}$ & $\mathrm{Cr}$ & Emp & & & \\
\hline 1 & 2 & 35 & 1243 & 0 & 1 & 1279 & 0 & 6 & 1274 & & & \\
\hline 2 & 217 & 419 & 644 & 2 & 111 & 1167 & 4 & 219 & 1057 & & & \\
\hline 3 & 1062 & 185 & 33 & 231 & 680 & 369 & 231 & 822 & 227 & & & \\
\hline \multirow[t]{3}{*}{4} & 1279 & 1 & 0 & 1047 & 228 & 228 & 1045 & 233 & 2 & & & \\
\hline & \multicolumn{3}{|c|}{$\varsigma=1,2$} & \multicolumn{3}{|c|}{$s=1,5$} & \multicolumn{3}{|c|}{$\varsigma=\mathbf{2 , 0}$} & \multicolumn{3}{|c|}{$\zeta=2,5$} \\
\hline & $\mathrm{Fe}$ & $\mathrm{Cr}$ & Emp & $\mathrm{Fe}$ & $\mathrm{Cr}$ & Emp & $\mathrm{Fe}$ & Crr & Emp & $\mathrm{Fe}$ & $\mathrm{C}$ & Emp \\
\hline 1 & 0 & 5 & 1275 & 0 & 34 & 1246 & 0 & 2 & 1278 & 0 & 21 & 1259 \\
\hline 2 & 3 & 358 & 919 & 2 & 646 & 632 & 0 & 200 & 1080 & 0 & 643 & 637 \\
\hline 3 & 212 & 943 & 125 & 230 & 1008 & 42 & 4 & 1075 & 201 & 7 & 1249 & 24 \\
\hline 4 & 1066 & 213 & 1 & 1049 & 231 & 0 & 214 & 1065 & 1 & 212 & 1051 & 0 \\
\hline 5 & 1279 & 1 & 0 & 1279 & 1 & 0 & 1063 & 217 & 0 & 1045 & 235 & 0 \\
\hline 6 & & & & & & & 1279 & 1 & 0 & 1279 & 1 & 0 \\
\hline
\end{tabular}

one layer to the next layer occurs with equal probability to any of the nearest-neighbor empty sites. Two variants of the algorithm were applied. Within the first (a) variant the atoms are forced to move on to one of the available (empty) sites, in the second (b) an atom can stop its descent with definite probability even if not all the nearest places in the next layer are already filled. Obviously, the second variant will lead to a rougher surface.

To simulate $\mathrm{Cr}$ overlayers on the $\mathrm{Fe}$ substrate we first distribute 320 atoms of Fe using both variants of the epitaxy algorithm. For dense packing this corresponds to five layers. This is sufficient for reproducing self-consistently the bulk moments in the lower layers. After all the $\mathrm{Fe}$ atoms have been added, $s \times 64$ ( $s$ is the coverage parameter) $\mathrm{Cr}$ atoms are added to the top of the prism using one of the two algorithms. The structure of the interface as obtained from variants $a$ and $b$ of "epitaxy" is given in Tables I and II. These tables show the number of $\mathrm{Fe}$ and $\mathrm{Cr}$ atoms as well as the number of empty sites in each layer, beginning from the surface. The results in Table I were modeled by using the $a$ variant for the Fe substrate, while the results in Table II were obtained from the $b$ algorithm for the Fe substrate to simulate interface alloying. In both cases the $a$ algorithm was used for modeling the $\mathrm{Cr}$ overlayer. As a result we obtain bcc lattices with sites occupied either by $\mathrm{Fe}$ or $\mathrm{Cr}$ atoms or vacant. For each of these structures, we determine selfconsistently the local magnetic moment at every site. Modeling of the sample and the self-consistent calculation of the magnetic moment distribution were repeated 20 times to effectively average over a larger sample. Calculations of the magnetic moment distribution were performed within a periodic Anderson model by a recursive method in real space. $^{20,21}$ The mass operator was calculated taking into account $d$ - $d$ interaction inside one coordination sphere of the atom under consideration. ${ }^{22,23}$

Since the purpose of our discussion is to model the magnetic structure of real interface, the magnetic moments are different not only between different layers, but also within

TABLE II. Layer by layer distribution of $\mathrm{Fe}$ atoms, $\mathrm{Cr}$ atoms, and empty sites for the set of rough structures generated by combination of $a$ and $b$ variants of algorithm "epitaxy" for different coverages.

\begin{tabular}{|c|c|c|c|c|c|c|c|c|c|c|c|c|}
\hline & \multicolumn{3}{|c|}{$s=0,5$} & \multicolumn{3}{|c|}{$s=1,5$} & \multicolumn{3}{|c|}{$s=2,0$} & \multicolumn{3}{|c|}{$\varsigma=\mathbf{3 , 0}$} \\
\hline & $\mathrm{Fe}$ & $\mathrm{Cr}$ & Emp & $\mathrm{Fe}$ & $\mathrm{Cr}$ & Emp & $\mathrm{Fe}$ & $\mathrm{Cr}$ & Emp & $\mathrm{Fe}$ & $\mathrm{Cr}$ & Emp \\
\hline 1 & 2 & 0 & 1278 & 2 & 5 & 1273 & 1 & 20 & 1259 & 0 & 6 & 1274 \\
\hline 2 & 25 & 3 & 1252 & 28 & 59 & 1193 & 21 & 153 & 1106 & 0 & 55 & 1225 \\
\hline 3 & 163 & 300 & 1087 & 168 & 272 & 840 & 158 & 464 & 658 & 2 & 260 & 1018 \\
\hline 4 & 467 & 98 & 715 & 480 & 496 & 304 & 467 & 646 & 167 & 17 & 608 & 655 \\
\hline 5 & 771 & 143 & 366 & 795 & 377 & 108 & 761 & 500 & 19 & 141 & 885 & 264 \\
\hline 6 & 976 & 105 & 199 & 958 & 253 & 69 & 957 & 291 & 32 & 464 & 783 & 33 \\
\hline 7 & 1030 & 82 & 168 & 1022 & 177 & 81 & 1032 & 185 & 63 & 786 & 488 & 6 \\
\hline 8 & 1033 & 72 & 175 & 1043 & 122 & 115 & 1045 & 129 & 106 & 971 & 286 & 23 \\
\hline 9 & 1066 & 51 & 163 & 1061 & 80 & 139 & 1061 & 91 & 128 & 1031 & 172 & 77 \\
\hline 10 & 1059 & 30 & 191 & 1043 & 50 & 187 & 1071 & 57 & 152 & 1035 & 126 & 119 \\
\hline 11 & 1088 & 26 & 166 & 1080 & 29 & 171 & 1106 & 24 & 158 & 1047 & 92 & 141 \\
\hline 12 & & & & & & & & & & 1066 & 57 & 157 \\
\hline 13 & & & & & & & & & & 1120 & 22 & 138 \\
\hline
\end{tabular}




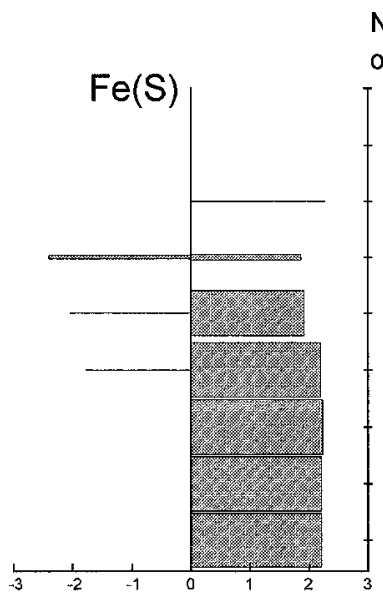

\section{Number}
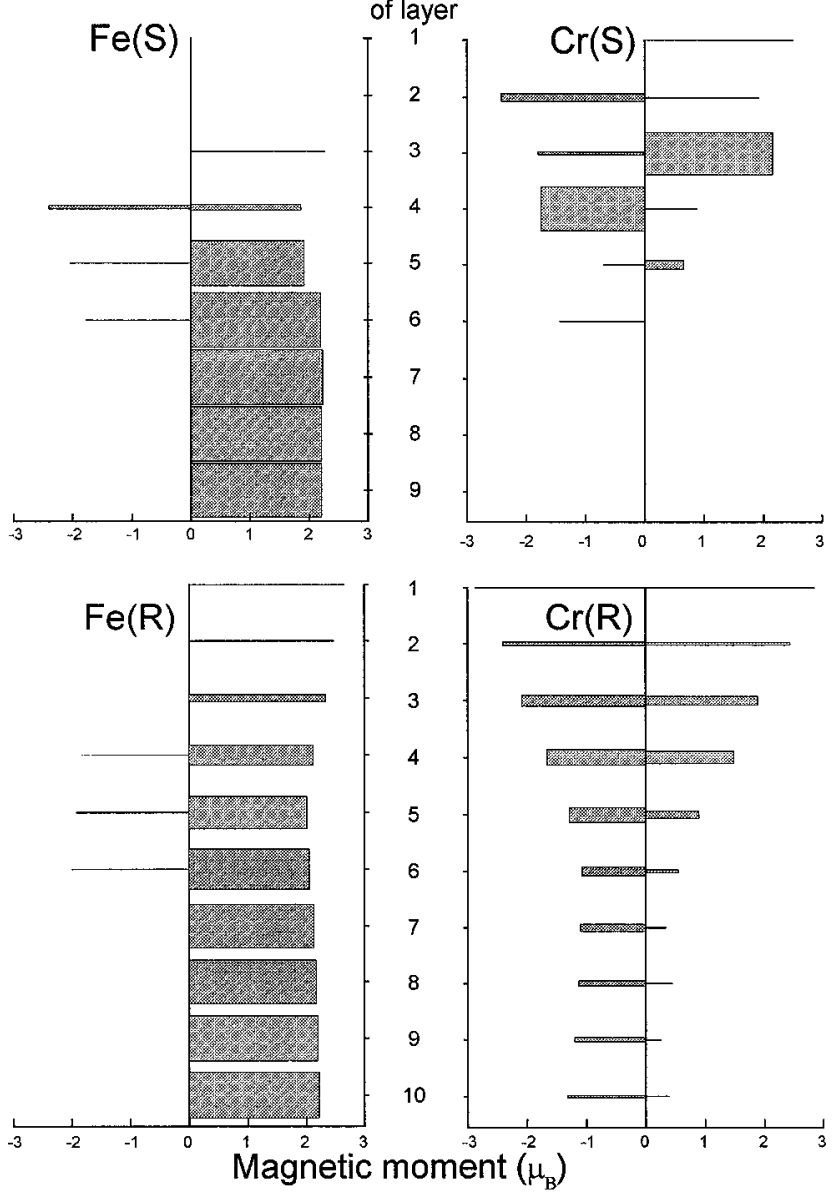

FIG. 2. Layer by layer distribution of magnetic moments on Fe and $\mathrm{Cr}$ atoms for the smooth $(S)$ and rough $(R)$ interface. Cr coverage $s=2 \mathrm{ML}$.

one layer there can be a significant variation of the magnetic moment because of the strongly varying surroundings of individual atoms. To illustrate the general trend of the magnetic moments in different layers, we performed an averaging for all subsets of $\mathrm{Cr}$ and $\mathrm{Fe}$ atoms within one layer and one spin orientation. The result is shown in Fig. 2 for 2 ML $\mathrm{Cr}$ on $\mathrm{Fe}$, modeled either as a smooth (upper two panels) or as a rough interface (lower). The lengths of the bars indicate the average magnetic moment in a particular layer, where the layer numbering is as in Tables I and II. The widths of the bars indicate the numbers of atoms with spin orientation parallel (plotted to the right, positive moment) or antiparallel to that of the Fe majority-spin orientation. Therefore the area of the bars represents the total magnetic moment in a given layer oriented either ferro- or antiferromagnetically with respect to that of Fe. The spacing of the bars is chosen equal to the width for $100 \%$ of atoms in one subset. The variation of the bar widths and appearance of the empty spaces between bars come about from atoms with opposite spin orientation, sites being occupied by the other atomic species, or vacant sites.

Figure 2 shows that the smooth interface has a behavior similar to that of an ideal interface: The magnetic moment in the top Fe layer is reduced compared to the bulk, and the $\mathrm{Cr}$ moment in the interface layer is antiparallel that of Fe. The two successive $\mathrm{Cr}$ layers are oriented antiferromagnetically, consistent with the layered antiferromagnetic (AF) structure of bulk Cr. The amount of intermixing is fairly limited, with some $\mathrm{Fe}$ incorporated in the first $\mathrm{Cr}$ layer, and a smaller amount of $\mathrm{Cr}$ in the top $\mathrm{Fe}$ layer. For these $\mathrm{Cr}$ atoms there is a ferromagnetic alignment with respect to $\mathrm{Fe}$, probably as a result of the dominating interaction with the $\mathrm{Cr}$ in the adlayer. The $\mathrm{Cr}$ moment in both layers is strongly enhanced compared to the bulk, but not as much as has been reported for a single $\mathrm{Cr}$ monolayer on $\mathrm{Fe}$. A few $\mathrm{Cr}$ atoms occupy already sites in the top layer, and these have an even larger moment of about $2.5 \mu_{B}$. This can be related to the low coordination number of these atoms associated with the small number of atoms in this layer. For all Cr layers, we find also some atoms with magnetic moment antiparallel to that of the ideal interface. These moments are smaller than those with orientation parallel to the "ideal" one, which we ascribe to increased frustration.

For the rough interface (lower two panels, Fig. 2) the situation is quite different. First we note that even down to the fifth substrate layer from the interface only about $80 \%$ of the sites are occupied by $\mathrm{Fe}$ with majority-spin orientation. Also, intermixing leads to $\mathrm{Cr}$ atoms being present down to this layer (about 2\%, see Table II). Furthermore, there is quite a significant number of empty sites. The $\mathrm{Cr}$ moments of about $1.3-1.5 \mu_{B}$ are largely antiparallel to those of Fe. Magnetic moments parallel to $\mathrm{Fe}$ are also found, but again of much smaller magnitude. The ferromagnetic alignment is caused, e.g., by $\mathrm{AF}$ interaction between $\mathrm{Cr}-\mathrm{Cr}$ nearest neighbors which are quite abundant even for $\mathrm{Cr}$ concentrations of a few $\%$, in competition with the $\mathrm{AF} \mathrm{Cr}-\mathrm{Fe}$ interaction, with the concomitant frustration leading to a reduction of the magnetic moment. At the interface, the intermixing is much stronger, to an extent that the interface is hardly recognizeable any more, at least in the $\mathrm{Cr}$ distribution. In the four topmost $\mathrm{Cr}$ layers the ferro- and antiferromagnetically aligned magnetic moments are of similar magnitude, with the antiferromagnetic moments slightly winning out close to the nominal interface. Taking into account also the relative abundance, the antiferromagnetic alignment dominates for the layers close to the interface, while the topmost layers have a vanishing net moment.

We will now use this type of model to discuss experimental core-level photoemission data which provide information on the magnetic order of the overlayer either from spin polarization or from magnetic dichroism. In the context of the present discussion, we will suppose that the electron spin polarization in a core-level photoemission spectrum or the asymmetry in magnetic linear dichroism are proportional to the value of the local magnetic moment. ${ }^{24-26}$ In our discussion we will refer to the spin polarization, however, the same formulas can be used for analyzing dichroism spectra. For an ideally smooth surface all the atoms in one atomic layer have the same magnetic moment, such that the resulting spin polarization can be written in the following way:

$$
f>M_{1}^{S}+\alpha M_{2}^{S}+\alpha^{2} M_{3}^{S}+\cdots .
$$

$M_{i}^{S}$ is local magnetic moment of the atoms of type $S$ ( $S$ $=\mathrm{Fe}$ or $\mathrm{Cr}$ ) from the $i$ th layer, and $\alpha$ takes into account the attenuation of the photoelectron signal arising from nonsurface layers due to the finite mean free path. As a simple 
approach $\alpha$ can be expressed through a universal escape depth $\lambda$, which depends on the kinetic energy of the photoelectrons, and a characteristic length $L$, which is the spacing of lattice planes parallel to the surface

$$
\alpha=\exp (-L \cos \theta / \lambda),
$$

where $\theta$ is the emission angle referred to the surface normal. For real surfaces and interfaces with roughness, expressions (1) and (2) have to be modified for two reasons. Firstly, the local magnetic moment depends not only on the distance of the atom from the surface, but also on its local environment, i.e., the number of $\mathrm{Fe}$ and/or $\mathrm{Cr}$ neighboring atoms as well as their magnetic states. Secondly, the probability of electron scattering is determined not only by the number of the layers which the photoelectrons has to traverse (i.e., distance from the surface), but also by the structure of the rough surface. For example, for a stepped (100) surface atoms that are in the different layers can still be surface atoms. If the surface has local defects such as atomic scale holes or islands, this will also affect the effective value of $\alpha$.

To determine the polarization of photoelectron $f_{i}$ emitted from the $i$ th layer, let us consider the top layer of the sample. All the atoms of this layer are surface atoms and consequently

$$
f_{1}>M_{1}^{S},
$$

where $M_{1}^{S}$ is the total magnetic moment of the $S$ atoms in the top layer. For the second layer, in which some atoms are covered by atoms of the first layer, whereas others are not covered and are still surface atoms, we have

$$
f_{2}>M_{2}^{S}\left[\alpha \frac{N_{1}}{N}+\left(1-\frac{N_{1}}{N}\right)\right]=M_{2}^{S}\left[1+(\alpha-1) \frac{N_{1}}{N}\right],
$$

where $N_{1}$ is the number of atoms in the 1 layer (not dependent on the kind of atoms), $N$ is the total number of the places in this layer.

Analogously, we have for the third layer

$$
\begin{aligned}
f_{3}> & M_{3}^{S}\left\{\alpha^{2} \frac{N_{1}}{N} \frac{N_{2}}{N}+\alpha\left[\left(1-\frac{N_{1}}{N}\right) \frac{N_{2}}{N}+\left(1-\frac{N_{2}}{N}\right) \frac{N_{2}}{N}\right]\right. \\
& \left.+\left(1-\frac{N_{1}}{N}\right)\left(1-\frac{N_{2}}{N}\right)\right\} .
\end{aligned}
$$

The first term in the figure bracket corresponds to transfer of an electron through two filled layers above the emitting layer, and the second term describes the situation of a photoelectron encountering an empty site in the top layer; the third term corresponds to the case when the sites both in layers 1 and 2 are empty, and effectively some atoms of the third layer appear to be surface atoms. After simple reducing for $f_{3}$ we have

$$
f_{3}>M_{3}^{S}\left[1+(\alpha-1) \frac{N_{1}}{N}\right]\left[1+(\alpha-1) \frac{N_{2}}{N}\right] .
$$

In the general case one can obtain the following recurrent expressions for the contribution to the polarization from the $j+1$ layer:
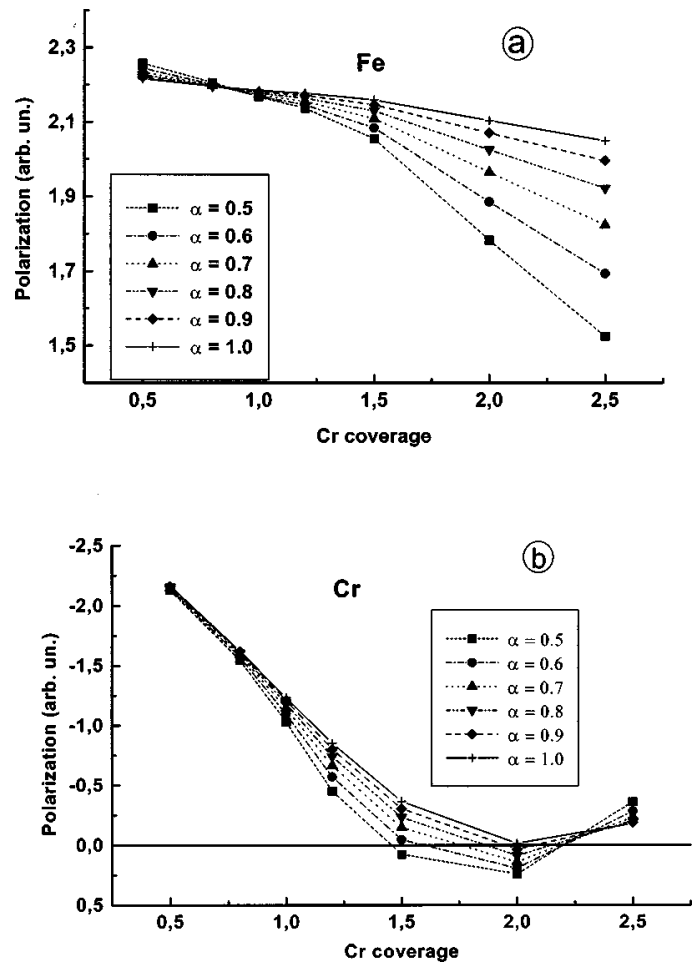

FIG. 3. Coverage dependence of polarization on $\mathrm{Fe}$ (a) and $\mathrm{Cr}$ (b) atoms for the samples with a "smooth"' interface generated by the $a$ variant of algorithm "epitaxy." Different symbols correspond to various values of the parameter $\alpha$.

$$
\frac{M_{j}^{S}}{M_{j+1}^{S}} f_{j+1}=f_{j} \frac{N_{j}}{N} \alpha+f_{j}\left(1-\frac{N_{j}}{N}\right)=f_{j}\left[1+(\alpha-1) \frac{N_{j}}{N}\right] .
$$

As a result for total polarization $F=\sum_{i=1}^{\infty} f_{i}$ we obtain

$$
F=\sum_{i=1}^{\infty} M_{i}^{S} \prod_{j=1}^{i-1}\left[1+(\alpha-1) \frac{N_{j}}{N}\right]
$$

In spectroscopy, usually the spin polarization is measured, which is given by the normalized difference between the number of electrons with spin-up and spin-down projection $\left(I_{ \pm}\right)$emitted from the surface as

$$
I=\frac{I_{+}-I_{-}}{I_{+}+I_{-}} .
$$

To obtain such a normalized polarization, we have to multiply $F$ by the factor $Z$ :

$$
Z^{-1}=\sum_{i=1}^{\infty} N_{i}^{S} \prod_{j=1}^{i-1}\left[1+(\alpha-1) \frac{N_{j}}{N}\right],
$$

which takes into account the reduction of the number of emitted electrons with decreasing concentration of that atomic species. Note, that $I=Z F$ for the $\mathrm{Cr}$ atoms does not vanish when the coverage parameter $s$ approaches zero.

\section{DISCUSSION}

Figures 3 and 4 show the spin polarization calculated as described above for two sets of overlayers as a function of 

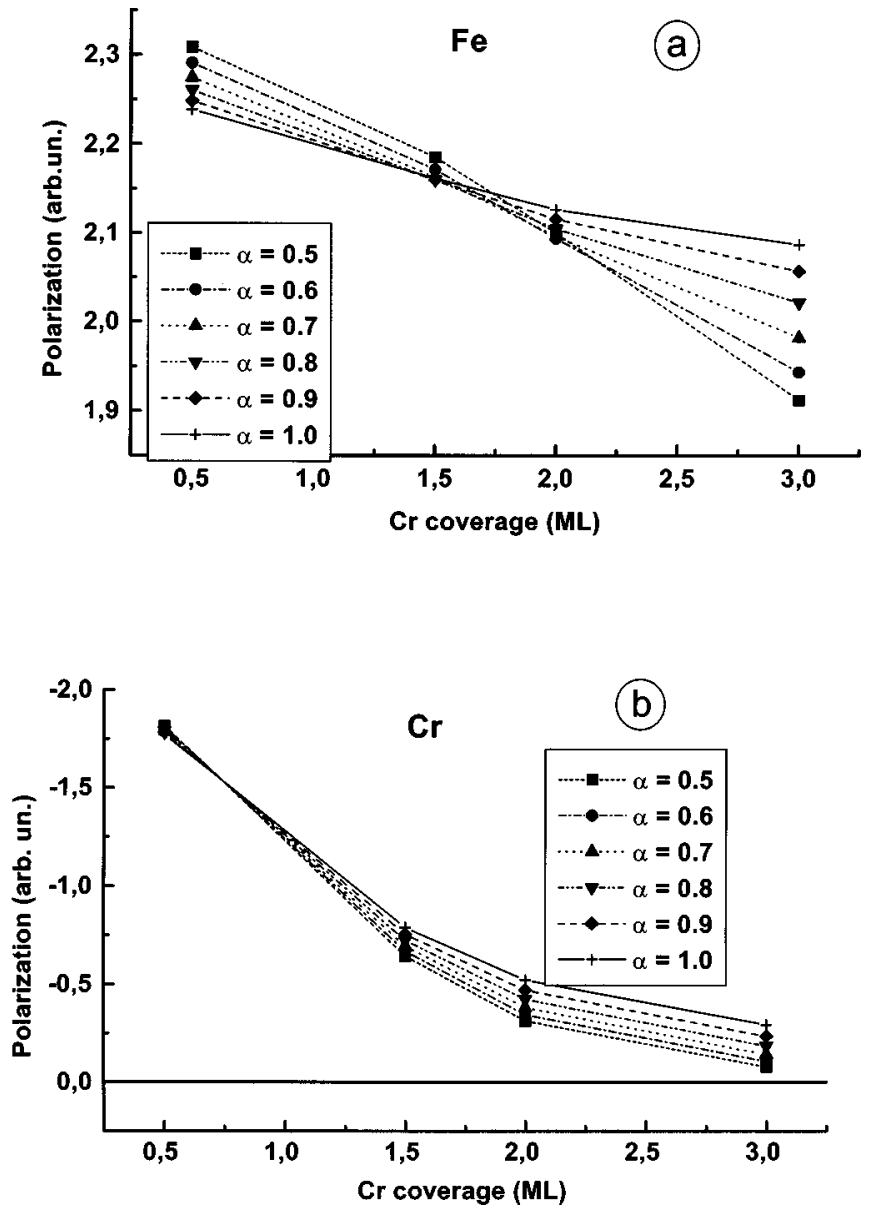

FIG. 4. Coverage dependence of polarization on $\mathrm{Fe}$ (a) and $\mathrm{Cr}$ (b) atoms for the samples with a "rough" interface generated by combination of $a$ and $b$ variants of algorithm "epitaxy." Different symbols correspond to various values of the parameter $\alpha$.

the $\mathrm{Cr}$ coverage. The results correspond to relatively smooth and rough surfaces, respectively, as generated by variants $a$ and $b$ of our epitaxy algorithm. All magnetic moments for these spatially inhomogeneous systems were calculated selfconsistently, and the Fermi level was chosen so that the total number of $d$ electrons in the system remained constant. Different symbols in Figs. 3 and 4 correspond to various values of the escape length parameter $\alpha$. We point out, however, that the general tendency of the expected spin polarization or magnetic dichroism within this model does not depend on the specific value of this parameter.

We note first that the magnetic signal of the Fe decreases monotonically with coverage s. This is connected with the decrease of the $\mathrm{Fe}$ magnetic moments under the action of $\mathrm{Cr}$ neighbors. The higher the $\mathrm{Cr}$ coverage, the more $\mathrm{Fe}$ atoms have a reduced magnetic moment. We assume further that additional scattering of photoelectrons by the $\mathrm{Cr}$ overlayer atoms is spin independent and only leads to a decrease of the number of photoelectrons, but not to a change of the normalized polarization (3). The decrease of the polarization with $s$ is more rapid for smaller escape depth $\lambda$ (small $\alpha$ ). The strongest decrease of $\mathrm{Fe}$ magnetic moments under the action of $\mathrm{Cr}$ neighbors takes place for the surface atoms, whose moments are enhanced for the free surface. Small $\lambda$ leads a smaller contribution of inner Fe atoms whose magnetic moments change only a little with $\mathrm{Cr}$ coverage.
For low $\mathrm{Cr}$ coverages, e.g., for $\boldsymbol{\varsigma} \approx 0.5$, the polarization is smaller for larger $\alpha$. However, for this value of $\alpha$ it decreases with $\varsigma$ more slowly. As a result there is a distinct coverage $\varsigma_{0}$ for which the spin polarization does not depend on $\alpha$. For a smooth interface as in Fig. 3(a) this takes place for $s_{0}$ of the order 0.5-1 ML, whereas for rough interfaces as in Fig. 4(a) $\varsigma_{0}$ is of the order of 1.5-2 ML. If $\alpha$ is changed by changing the takeoff angle $\theta$ between the sample surface and the direction of the electron beam, there will be no dependence of the magnetic signal on this angle at this coverage. As is seen from Figs. 3 and 4, this specific coverage depends on the surface roughness. Actually we propose that value of this specific coverage can be used to characterize the amount of interface roughness. Note that the $\mathrm{Cr}$ signal for the same coverage has another dependence on $\alpha$.

The polarization of the $\mathrm{Cr}$ signal may oscillate or decrease monotonically with $s$ depending on the surface roughness and on the interdiffusion in the interface region. For $\mathrm{Cr}$, there is a general decrease of the polarization which is much faster than for the Fe substrate. This is related to antiferromagnetic coupling between $\mathrm{Cr}$ atoms. The dependence of the $\mathrm{Cr}$ polarization on the escape depth is analogous to that found for the Fe substrate: a small $\alpha$ leads to a more rapid decrease of the polarization with coverage. This is in part caused by a transfer of $\mathrm{Cr}$ atoms through the $\mathrm{Fe}$ surface to vacant $\mathrm{Fe}$ sites. For such $\mathrm{Cr}$ atoms embedded in the $\mathrm{Fe}$ matrix we find a moment opposite to $\mathrm{Fe}$, and consequently there is a negative contribution to the spin polarization. The role of such inner atoms is reduced with a decrease of the escape depth parameter. Subsurface $\mathrm{Cr}$ atoms for rough surfaces have magnetic moments, ordered in both directions and their contributions cancel much faster with increasing Cr coverage.

If oscillatory behavior does occur, the oscillations are more pronounced for small $\alpha$. The maximum of the polarization is obtained for $2 \mathrm{ML}$ coverage when the polarization has the same sign as that of the Fe substrate. For ideally smooth surfaces this behavior is quite natural. The first $\mathrm{Cr}$ monolayer on $\mathrm{Fe}$ has a surface-enhanced moment opposite to the Fe moments. When the next ideally smooth Cr layer is deposited, it will have a surface-enhanced moment opposite to the previous one. Furthermore it will reduce the value of the magnetic moment of the previous layer, because those atoms are not at the surface any more. This leads to a change of the sign of the polarization with every additional monolayer. Roughness will erode such an oscillation but as can be seen from Fig. 3(b) it does not destroy oscillations for relatively smooth surfaces. For rough interfaces this signature of the antiferromagnetic structure is fully destroyed [Fig. 4(b)].

Turning now to experimental data, Fig. 5 shows the spin polarization and the magnetic linear dichroism obtained in photoemission experiments for $\mathrm{Cr}$ films grown epitaxially on Fe substrates. The common observation in all the experiments is an antiferromagnetic alignment of the $\mathrm{Fe}$ and $\mathrm{Cr}$ magnetic moments. Spin-polarized photoemission data are shown for the Fe $3 p$ and $\mathrm{Cr} 3 p$ core-level spectra as a function of $\mathrm{Cr}$ coverage for several $\mathrm{Cr}$ films on an $\mathrm{Fe}$ (100) thinfilm substrate. From low-energy electron diffraction investigations of the Fe films it had been concluded that the films grown on $\mathrm{Cu}_{3} \mathrm{Au}(100)$ were more disordered than those on the $\operatorname{Ag}(100)$ substrate. $^{2}$ The magnetic linear dichroism for the $\mathrm{Cr} 2 p$ level shows a weak indication of an oscillatory 


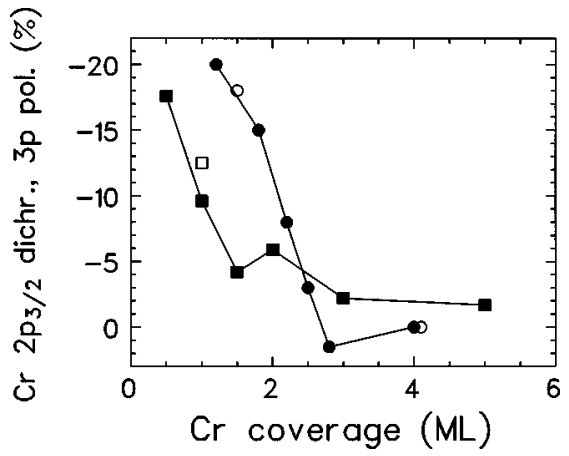

FIG. 5. Experimental spin-polarization in $\mathrm{Cr} 3 p$ photoemission spectra as function of $\mathrm{Cr}$ coverage for $\mathrm{Cr} / \mathrm{Fe} / \mathrm{Cu}_{3} \mathrm{Au}(100)$ (filled circles) and $\mathrm{Cr} / \mathrm{Fe} / \mathrm{Ag}(100)$ (empty circles); $\mathrm{Cr} 2 p$ magnetic linear dichroism for $\mathrm{Cr} / \mathrm{Fe} / \mathrm{Ag}(100)$ (filled squares) and $\mathrm{Cr} / \mathrm{Fe} / \mathrm{W}(110)$ (empty square).

dependence — albeit without a change of sign —of the dichroism on the $\mathrm{Cr}$ coverage.

Comparison of the experimental and theoretical curves suggests some conclusions about the microscopic structure of the $\mathrm{Cr}$ overlayer. For smooth surfaces, the theoretical model predicts a monotonous change of the spin polarization up to two monolayers $\mathrm{Cr}$ coverage, and even a change of spin polarization near this point. The dichroism experiment, in contrast, shows an oscillation and a maximum instead of a minimum for two monolayers coverage. This can be explained if one supposes a nonuniform growth of the second layer, which leads to an overlayer consisting of patches with 1 and 3 monolayer coverage, while the occurrence of $2 \mathrm{ML}$ coverage is suppressed. This will increase the absolute value of the dichroism for the $\mathrm{Cr}$ signal. Further deposition of $\mathrm{Cr}$ leads to the filling of the space around islands, which decreases the dichroism.

The behavior suggested here is consistent with a large part of the presently available experimental data. Pierce et $a l .{ }^{16}$ found that there is a "defect" in the antiferromagnetic ordering between 1 and 4 layers coverage giving a phase change in electron-spin polarization $P(\mathrm{Cr})$, although for the thicker coverage they were able to observe oscillations of $P(\mathrm{Cr})$ as a function of $\mathrm{Cr}$ thickness with a period of two atomic layers. Idzerda et al. ${ }^{15}$ found a monotonous decrease of the X-ray magnetic circular dichroism (XMCD) signal with $\mathrm{Cr}$ thickness for $\mathrm{Cr}$ overlayers on Fe. Böske et al. ${ }^{27}$ found for the XMCD signal of $2 \mathrm{ML} \mathrm{Cr}$ on $\mathrm{Fe}(100)$ the same sign as for $1 \mathrm{ML}$, contrary to the simple model of layer-bylayer growth. They connected such behavior to a special three-dimensional island growth. Turtur and Bayreuther ${ }^{3}$ found a rapid decrease of the total magnetic moment of an $\mathrm{Fe}$ film sample on deposition of a $\mathrm{Cr}$ overlayer. This led them to the suggestion that the first two $\mathrm{Cr}$ layers on Fe have in fact parallel magnetic moments opposite to the magnetic moment of Fe substrate. The common feature of the results is that in the low coverage regime the distribution of thicknesses is not Poisson-like. $^{15}$

For explaining these experiments, where a non-Poisson three-dimensional growth of the $\mathrm{Cr}$ islands on $\mathrm{Fe}$ was clearly revealed, we will put forward a simple theory based on the idea of confinement of itinerant electrons within the $\mathrm{Cr}$ islands on Fe surface. The existence of confined quantum-well (QW) states for electrons in highly perfect layer structures is well established both experimentally ${ }^{28}$ and theoretically. ${ }^{29}$ For Fe/Cr multilayers, spin-polarized QW-like states were analyzed within the framework of $a b$ initio calculations ${ }^{29}$ and it was shown that a QW-model yields a better description of the oscillatory exchange coupling in iron-chromium systems than Ruderman-Kittel-Kasuya-Yosida-like models. Recently quantum-size effects were considered as the origin of the formation of needlelike metallic islands on the surfaces. ${ }^{30}$

Let us consider a $\mathrm{Cr}$ island with thickness $L$ on an ideal $\mathrm{Fe}$ surface, and further suppose that at least electrons with one spin projection are fully confined within the island. If $L$ is much less than the area of the island, we can use an infinite QW model for describing the transverse movement of the confined electrons, and a free-electron approach for the electron movement in plane. In this case the density of states will have the form

$$
\rho(\omega)=\frac{m S}{(2 \pi \hbar)^{2}} \sum_{n} \theta\left(\omega-\varepsilon_{n}\right),
$$

where $\varepsilon_{n}=\pi^{2} \hbar^{2} n^{2} / 2 m^{2} L^{2}$ is the energies of electrons in $\mathrm{QW}, S$ is the area of island.

We assume that the Fermi energy of the system is fixed by the large number of electrons in the Fe substrate. In this case the total number of confined electrons is not fixed. To compare the states with different distribution of island thicknesses we have to consider the thermodynamic potential $\Omega$ $=E-\varepsilon_{F} N$ of the electrons in all QW's. For the single well we have

$$
\begin{aligned}
\Omega_{L}= & \frac{S m \varepsilon_{F}^{2} n_{L}}{4 \pi \hbar^{2}}\left\{-1+\frac{1}{3}\left(\frac{\pi^{2} \hbar^{2}}{2 m \varepsilon_{F} L^{2}}\right)\left(n_{L}+1\right)\left(2 n_{L}+1\right)\right. \\
& \left.\times\left[1-\left(\frac{\pi^{2} \hbar^{2}}{2 m \varepsilon_{F} L^{2}}\right) \frac{3 n_{L}^{2}+3 n_{L}-1}{10}\right]\right\}
\end{aligned}
$$

Here $n_{L}$ is the number of quantum levels below the Fermi energy. The analysis of this problem can be significantly simplified if instead of $\Omega_{L}$ we consider the quantity $\Delta \Omega_{L}$ $=\Omega_{L}-\Omega_{L}^{\mathrm{cl}}$, where $\Omega_{L}^{\mathrm{cl}}$ is the quasiclassical contribution, which can be obtained from Eq. (4) by substitution of the integer number $n_{L}$ by its quasiclassical value $\alpha$ :

$$
n_{L} \rightarrow \alpha=\frac{\sqrt{2 m \varepsilon_{F}}}{\hbar \pi} L .
$$

A straightforward calculation shows that $\Omega_{L}^{\mathrm{cl}}$ contains only contributions proportional to the $L^{1}, L^{0}, L^{-3}$ :

$$
\Omega_{L}^{\mathrm{cl}}=\frac{S m \varepsilon_{F}^{2}}{4 \pi \hbar^{2}}\left[-\frac{8}{15} \alpha+\frac{1}{2}+\frac{1}{30} \alpha^{-3}\right] .
$$

After summation of the contribution to $\Omega^{\mathrm{cl}}$ over all the QW's on the sample surface, we will obtain the term proportional to the total volume of all islands (which is constant for the given coverage parameter s), and a term proportional to the total area of the islands (also constant for a coverage exceeding one monolayer). The third contribution in Eq. (5) appears to be small even for monolayer islands and can be omitted. As a result, when the coverage $s$ is fixed, for the determination of the distribution of islands on thickness it is enough to compare $\Sigma_{L_{i}} \Delta \Omega_{L_{i}}$ instead of $\Sigma_{L_{i}} \Omega_{L_{i}}$. 


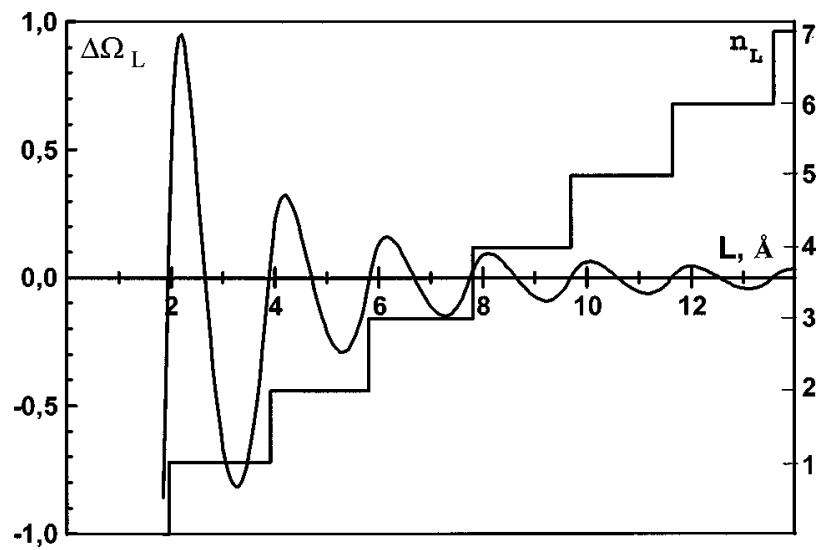

FIG. 6. $\Delta \Omega_{L}$ (a.u.) and number of quantum levels in the well versus $L$.

In Fig. 6, the dependence of $\Delta \Omega_{L}$ for the single QW and the number of quantum levels in the well versus their width $L$ is shown. $\Delta \Omega_{L}$ oscillates with $L$ and decreases as $L^{-2}$. This means that the main contribution to the $\Sigma_{L_{i}} \Delta \Omega_{L_{i}}$ arises from electrons localized in the narrowest $\mathrm{QW}$, much as the electrons in narrowest QW determine the oscillations of exchange coupling in metallic magnetic superlattices and sandwich systems. ${ }^{31}$ Note that for $\mathrm{Cr}$ islands on an $\mathrm{Fe}(100)$ surface, the thickness of the QW can be changed only discretely in steps of one half of the lattice constant. The period of oscillation in Fig. 6 is about 2 A, i.e., of the same order as the lattice constant. Hence, if for the islands with thickness 2 $\mathrm{ML}, \Delta \Omega$ will be larger than for 1 and $3 \mathrm{ML}$, the formation of $2 \mathrm{ML}$ islands will be suppressed.

Our model of infinite QW's is too simple to explain the behavior of real systems in every detail. The finite depth and the shape of the QW have a strong influence on the phase and even period of the oscillation $\Delta \Omega_{L}$. Alloying in the interface region leads to erosion of the islands, so that our picture can be applied only for part of the sample surface. Despite these restrictions, confinement of the electrons in QW's definitely favors a non-Poisson distribution of island thicknesses. Clearly, the proposed model is only one of a number of conceivable mechanisms which may lead to the experimentally observed behavior. The growth of threedimensional islands for higher thicknesses reduces the dependence of polarization on coverage and leads to the same effect as alloying in the interface region. The suppression of $2 \mathrm{ML}$ islands can be one reason for the oscillation of the MCD signal which was obtained in our experiment as well as an apparent FM ordering of the first and the second $\mathrm{Cr}$ monolayer. m,27 $^{2}$

For a better understanding of the magnetic properties of the $\mathrm{Fe}-\mathrm{Cr}$ interface a better characterization of the interface is desirable. Soft X-ray reflectivity studies can be very helpful for assessing the surface or interface roughness. Clearly, such studies are highly desirable for a system like $\mathrm{Cr}$ on $\mathrm{Fe}$, and we are sure will be carried out very soon. In combination with the soft x-ray Kerr effect, either by employing circularly polarized light or with linearly polarized light, in transverse geometry would even allow us to distinguish between chemical and magnetic roughness, which would be extremely useful in the present context. ${ }^{32}$

\section{ACKNOWLEDGMENTS}

This work was supported by Deutscher Akademischer Austauschdienst (Grant No. ARC 313) and INTAS (Grant No. 96-0531). Funding by the Bundesministerium für Forschung und Technologie (BMFT) under Grant No. 05621 PFA 7 as well as by the Deutsche Forschungsgemeinschaft (DFG) within Project No. SFB 166/G7 is gratefully acknowledged. V.M.U. would like to express his gratitude to the Alexander von Humboldt-Stiftung for financial support.
*Permanent address: St. Petersburg University, V.O. 14 Linia 29, 199178 St. Petersburg, Russia.

${ }^{1}$ C. Carbone and S. F. Alvarado, Phys. Rev. B 36, 1280 (1987).

${ }^{2}$ R. Jungblut, F. U. Hillebrecht, Ch. Roth, and E. Kisker, J. Appl. Phys. 70, 5923 (1991); F. U. Hillebrecht, Ch. Roth, R. Jungblut, E. Kisker, and A. Bringer, Europhys. Lett. 19, 711 (1992).

${ }^{3}$ M. Donath, D. Scholl, and D. Mauri, Phys. Rev. B 43, 13164 (1991).

${ }^{4}$ C. Turtur and G. Bayreuther, Phys. Rev. Lett. 72, 1557 (1994); S. Miethaner and G. Bayreuther, J. Magn. Magn. Mater. 148, 42 (1995).

${ }^{5}$ P. Fuchs, V. N. Petrov, K. Totland, and M. Landolt, Phys. Rev. B 54, 9304 (1996).

${ }^{6}$ B. Heinrich, J. F. Cochran, D. Venus, K. Totland, D. Atlan, S. Govorkov, and K. Myrtle, J. Appl. Phys. 79, 4518 (1996); D. Venus and B. Heinrich, Phys. Rev. B 53, R1733 (1996).

${ }^{7}$ R. Pfandzelter, T. Igel, and H. Winter, Phys. Rev. B 54, 4496 (1996); Phys. Rev. Lett. 76, 4175 (1996).

${ }^{8}$ L. Davies, J. A. Stroscio, D. T. Pierce, and R. J. Celotta, Phys. Rev. Lett. 76, 4175 (1996).

${ }^{9}$ J. Landes, Ch. Sauer, R. A. Brand, W. Zinn, and Zs. Kajcsos, Hyperfine Interact. 57, 1941 (1990).

${ }^{10}$ F. Klinkhammer, Ch. Sauer, E. Yu. Tsimbal, S. Handschuh, Q.
Leng, and W. Zinn, J. Magn. Magn. Mater. 161, 49 (1996).

${ }^{11}$ V. M. Uzdin and N. S. Yartseva, Comput. Mater. Sci. 10, 211 (1998).

${ }^{12}$ N. S. Yartseva, V. M. Uzdin, and C. Demangeat, Comput. Mater. Sci. 10, 255 (1998).

${ }^{13}$ N. Gittsovich, V. I. Minin, L. N. Romashev, V. G. Semenov, V. V. Ustinov, and V. M. Uzdin (unpublished).

${ }^{14}$ R. Jungblut, Ch. Roth, F. U. Hillebrecht, and E. Kisker, Surf. Sci. 269/270, 615 (1992).

${ }^{15}$ Y. U. Idzerda, L. H. Tjeng, H.-J. Lin, G. Meigs, C. T. Chen, and J. Gutierrez, J. Appl. Phys. 73, 6204 (1993); Y. U. Idzerda, L. H. Tjeng, H.-J. Lin, J. Gutierrez, G. Meigs, and C. T. Chen, Phys. Rev. B 48, 4144 (1993).

${ }^{16}$ D. T. Pierce, R. J. Celotta, and J. Unguris, J. Appl. Phys. 73, 6201 (1993); J. Unguris, R. J. Celotta, and D. T. Pierce, Phys. Rev. Lett. 69, 1125 (1993).

${ }^{17}$ A. K. Kasansky and V. M. Uzdin, Phys. Rev. B 52, 9477 (1995).

${ }^{18}$ D. Knabben, Th. Koop, H. A. Dürr, F. U. Hillebrecht, and G. van der Laan, J. Electron Spectrosc. Relat. Phenom. 86, 201 (1997).

${ }^{19}$ V. M. Uzdin and C. Demangeat, J. Magn. Magn. Mater. 165, 458 (1997).

${ }^{20}$ H. Dreysse and C. Demangeat, Surf. Sci. Rep. 28, 65 (1997).

${ }^{21}$ C. Demangeat and V. M. Uzdin, J. Magn. Magn. Mater. 156, 202 (1996). 
${ }^{22}$ V. N. Gittsovich, V. G. Semenov, and V. M. Uzdin, J. Magn. Magn. Mater. 146, 165 (1995).

${ }^{23}$ M. S. Borczuch and V. M. Uzdin, J. Magn. Magn. Mater. 172, 110 (1997).

${ }^{24}$ Ch. Roth, F. U. Hillebrecht, H. B. Rose, and E. Kisker, Phys. Rev. Lett. 70, 3479 (1993); F. U. Hillebrecht, Ch. Roth, H. B. Rose, W. G. Park, E. Kisker, and N. A. Cherepkov, Phys. Rev. B 53, 12182 (1996).

${ }^{25}$ G. Panaccione, F. Sirotti, and G. Rossi, J. Electron Spectroscopy Relat. Phenom. 76, 189 (1995).

${ }^{26}$ R. Schellenberg, E. Kisker, M. Faust, A. Fanelsa, and F. U. Hillebrecht, Phys. Rev. B 58, 81 (1998).

${ }^{27}$ T. Böske, W. Clemens, D. Schmitz, J. Kojnok, M. Schäfer, V.
Cros, G. Y. Guo, and W. Eberhardt, Appl. Phys. A: Mater. Sci. Process. 61, 119 (1995).

${ }^{28}$ J. E. Ortega, F. J. Himpsel, G. J. Mankey, and R. F. Willis, Phys. Rev. B 47, 1540 (1993); J. E. Ortega and F. J. Himpsel, Phys. Rev. Lett. 69, 844 (1992); P. Segovia, E. G. Michel, and J. E. Ortega, ibid. 77, 3455 (1996).

${ }^{29}$ M. Schilfgaarde and W. A. Harrison, Phys. Rev. Lett. 71, 3870 (1993); C. Sommers and P. M. Levy, J. Phys. I 6, 1461 (1996).

${ }^{30}$ K. Jin, G. D. Mahan, H. Metiu, and Z. Zhang, Phys. Rev. Lett. 80, 1026 (1998).

${ }^{31}$ V. M. Uzdin and N. S. Yartseva, J. Magn. Magn. Mater. 156, 193 (1996); 165, 370 (1997).

${ }^{32}$ J. W. Freeland, V. Chakarian, K. Bussmann, and Y. U. Idzerda, J. Magn. Magn. Mater. (to be published). 